\title{
Dephasing in InAs/GaAs quantum dots
}

Borri, Paola; Langbein, Wolfgang Werner; Mørk, Jesper; Hvam, Jørn Märcher; Heinrichsdorff, F.; Mao, M.-H.; Bimberg, Dieter

Published in:

Physical Review B

Link to article, DOI:

10.1103/PhysRevB.60.7784

Publication date:

1999

Document Version

Publisher's PDF, also known as Version of record

Link back to DTU Orbit

Citation (APA):

Borri, P., Langbein, W. W., Mørk, J., Hvam, J. M., Heinrichsdorff, F., Mao, M-H., \& Bimberg, D. (1999).

Dephasing in InAs/GaAs quantum dots. Physical Review B, 60(11), 7784-7787.

https://doi.org/10.1103/PhysRevB.60.7784

\section{General rights}

Copyright and moral rights for the publications made accessible in the public portal are retained by the authors and/or other copyright owners and it is a condition of accessing publications that users recognise and abide by the legal requirements associated with these rights.

- Users may download and print one copy of any publication from the public portal for the purpose of private study or research.

- You may not further distribute the material or use it for any profit-making activity or commercial gain

- You may freely distribute the URL identifying the publication in the public portal

If you believe that this document breaches copyright please contact us providing details, and we will remove access to the work immediately and investigate your claim 


\title{
Dephasing in InAs/GaAs quantum dots
}

\author{
P. Borri, W. Langbein, J. Mørk, and J. M. Hvam \\ Research Center COM, The Technical University of Denmark, Building 349, DK-2800 Lyngby, Denmark \\ F. Heinrichsdorff, M.-H. Mao, and D. Bimberg \\ Institut für Festkörperphysik, Technische Universität Berlin, Hardenbergstrasse 36, 10623 Berlin, Germany
}

(Received 8 June 1999)

\begin{abstract}
The room-temperature dephasing in InAs/GaAs self-assembled quantum dots is measured using two independent methods: spectal-hole burning and four-wave mixing. Dephasing times weakly dependent on the excitation density are found, with a low density value of $290 \pm 80$ fs from spectal-hole burning and of 260 \pm 20 fs from four-wave mixing. [S0163-1829(99)00136-8]
\end{abstract}

One of the basic sources of information on light-matter interaction is optical line broadening. ${ }^{1}$ The line width of the absorption or emission spectrum of a given transition depends on both homogeneous and inhomogeneous broadening mechanisms. Different mechanisms such as phonon interaction, lifetime broadening, and carrier-carrier interaction can contribute to the homogeneous linewidth in semiconductors, as widely investigated in numerous structures in the past. One way to determine the homogeneous linewidth is to measure the time-resolved polarization decay, with the characteristic time $T_{2}$. The development of four-wave mixing techniques has allowed to measure $T_{2}$ in both bulk materials and low-dimensional heterostructures also in the presence of strong inhomogeneous broadening, where a simple linewidth analysis in the spectral domain fails. ${ }^{2}$ Recent breakthroughs ${ }^{3}$ in the growth of zero-dimensional semiconductor structures strengthen the interest in extracting the homogeneous linewidth of these systems., 4 "5specially the "phononbroadening" is controversial, because the discrete energy level structure is expected to reduce phonon interactions (phonon bottleneck). ${ }^{6}$ Moreover, the homogeneous linewidth of quantum dots (QD's) presents the intrinsic limit to their delta-functionlike density of states, which is the key property for the expected superior performance in applications such as quantum dot lasers. ${ }^{7}$ The presently best investigated QD systems are $\mathrm{In}(\mathrm{Ga}) \mathrm{As} / \mathrm{GaAs}$ self-organized quantum dots. These systems still show a significant inhomogeneous broadening due to their size distribution. ${ }^{3}$ Therefore, the homogeneous broadening can be measured by direct linewidth analysis only when a few dots are isolated from the ensemble. Scarce data only for low temperatures were reported until now. ${ }^{4}$ Four-wave mixing experiments on self-organized III-V QD's have not been reported so far, probably due to the weak signal that results from the small interaction volume and the large inhomogeneous broadening.

In this paper, we present the first measurements of the $T_{2}$ time for InAs/GaAs quantum dots at room temperature, using two independent methods: self-induced spectral holeburning (SI-SHB) and four-wave mixing (FWM). Both experiments are performed using optical pulses from the idler of an optical parametric amplifier, providing $140 \mathrm{fs}$ pulses at a repetition rate of $300 \mathrm{kHz}$ and a wavelength tunable from 0.9 to $2.5 \mu \mathrm{m}$. The pulses are dispersion compensated and can be spectrally shaped to get Fourier-limited pulse durations up to a few picoseconds.

The investigated sample was grown by metal organic chemical vapor deposition in a pin structure. Its active region consists of three stacked InAs/InGaAs QD layers separated by $21 \mathrm{~nm}$-thick GaAs barriers, placed in the center of a 120-nm GaAs layer. Two $\mathrm{Al}_{0.7} \mathrm{Ga}_{0.3} \mathrm{As}$ cladding layers and a ridge structure of $8 \mu \mathrm{m}$ width and $400 \mu \mathrm{m}$ length provide optical confinement and waveguiding. ${ }^{8}$ The end facets were tilted to avoid multiple reflections. The light is coupled in and out of the end facets of the waveguide by microscope objectives. Room-temperature photoluminescence of the QD ensemble after etching off the top cladding shows a groundstate transition at $1.148 \mathrm{eV}(1.08 \mu \mathrm{m})$ with a broadening of about $60 \mathrm{meV}$. The wetting layer transition in these binaryternary dots is closer in energy to the QD ground state as compared to pure InAs/GaAs dots, but the energy separation of $110 \mathrm{meV}$ is still significantly above $k_{\mathrm{B}} T$ at room temperature. ${ }^{8,9}$ The SI-SHB experiment uses the bleaching of the ground-state excitonic absorption induced by an optical pulse at $1.08 \mu \mathrm{m}$ wavelength traveling through the sample, resulting in an increased transmission of the pulse through the waveguide. The transmitted pulse is measured by a cooled Ge detector using a lock-in technique. The bleaching of the absorption with increasing input energy is shown in Fig. 1 for Fourier-limited pulses of different spectral widths and time durations. For short pulses, i.e., large spectral width, an increasing absorption is observed additional to the bleaching with increasing input energy, due to two-photon absorption. The $x$ axis gives the input spectral energy, i.e., the energy per unit frequency. For a given input spectral energy, the observed bleaching depends on the pulse width. We performed pulse shaping such that the input pulses are close to square shaped in the spectral domain. In order to interpret the results, we have calculated the spectral-hole burning of the absorption coefficient induced by a square pulse with a spectral energy $S$ and a spectral width $W$ larger or smaller than the full width at half maximum (FWHM) of the homogeneous broadening $\gamma=2 \hbar / T_{2}$ of the ground-state transition in a two-level model. The absorption coefficient $\alpha$ of an inhomogeneously broadened distribution in the presence of monochromatic pump light with the frequency $\omega_{\mathrm{p}}$ and the intensity $I_{\mathrm{p}}$ is given to first order in $I_{\mathrm{p}}$ by ${ }^{10}$ 


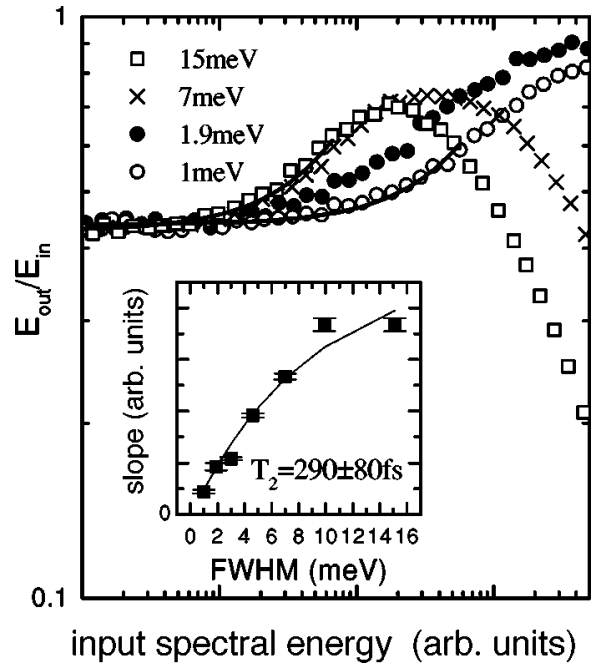

FIG. 1. QD transmission (ratio between the energy at the output and the energy at the input of the sample) in a logarithmic scale, as a function of the input spectral energy for different spectral pulsewidths. Solid lines are fits to the data according to the model described in the text [Eq. (4)]. In the inset, the slopes of the initial absorption bleaching and the corresponding fit are shown.

$$
\alpha\left(\omega, \omega_{\mathrm{p}}, I_{\mathrm{p}}\right)=\alpha_{0}\left[1-\frac{C T_{1} I_{\mathrm{p}}}{\left(\omega-\omega_{\mathrm{p}}\right)^{2}+\left(2 / T_{2}\right)^{2}}\right],
$$

$C$ is a constant containing the transition dipole moment, $T_{1}$ is the population decay time, and $\alpha_{0}$ is the unperturbed absorption coefficient with a spectral dependence given by the inhomogeneous broadening. Equation (1) describes SHB being proportional to the intensity, as expected for continuous-wave light. However, in the measurements of Fig. 1, pulses shorter than $T_{1}$ are always used. In this case, a population saturation, and therefore a spectral hole-burning, proportional to the pulse energy is expected. We replace the steady-state energy $T_{1} I_{\mathrm{p}}$ by the element of energy $S d w_{\mathrm{p}}$ and we sum Eq. (1) over the spectral components of a square pump pulse centered at $\omega_{0}$. This gives for the absorption:

$$
\begin{aligned}
\frac{\alpha(\omega, S, W)}{\alpha_{0}}= & 1-\frac{C S T_{2}}{2}\left\{\arctan \left[\left(\omega-\omega_{0}+W / 2\right) T_{2} / 2\right]\right. \\
& \left.-\arctan \left[\left(\omega-\omega_{0}-W / 2\right) T_{2} / 2\right]\right\}
\end{aligned}
$$

Since in our case the inhomogeneous broadening is much larger than $W$, we have assumed that $\alpha_{0}$ is constant over the pulse energy range. The result of Eq. (2) is shown in Fig. 2 for a pump centered at $\hbar \omega_{0}=1.15 \mathrm{eV}$ and $\gamma=1.1 \mathrm{meV}$. For $W \ll(\gamma / \hbar)$ a hole of a width $2 \gamma$ is burned in the absorption coefficient ${ }^{11}$ with a depth proportional to $W$ and $S$. In contrast, for $W \gg(\gamma / \hbar)$, a fingerprint of the square pulse is burned in the absorption coefficient with a depth proportional to $S$ and independent of $W$. The transmission of the pulse influenced by the self-induced bleaching is calculated from Eq. (2). In the limit of a small absorption $\alpha_{0} L<1$, where $L$ is the length of the waveguide, we find for the transmitted energy $E_{\text {out }}$ :

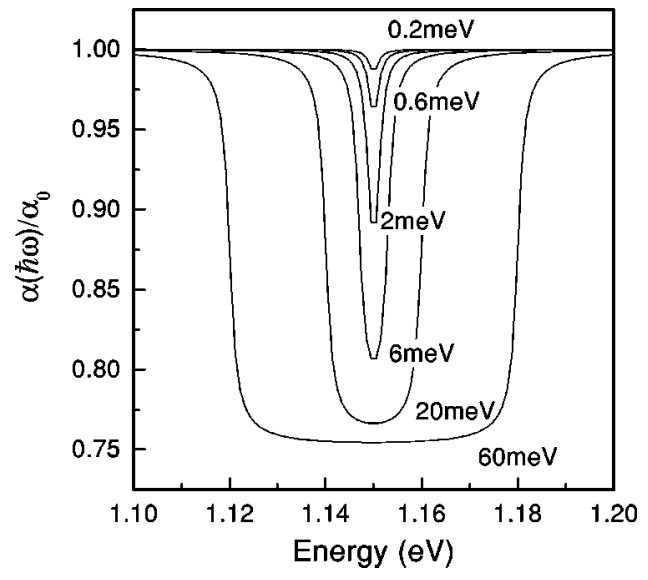

FIG. 2. Calculated absorption bleaching [Eq. (2)] for $\gamma$ $=1.1 \mathrm{meV}, \omega_{0}=1.15 \mathrm{eV}$, and different spectral pulse-widths as indicated.

$$
E_{\text {out }}=\int_{\omega_{0}-W / 2}^{\omega_{0}+W / 2} S[1-\alpha(\omega, S, W) L] d \omega
$$

$$
\begin{aligned}
\frac{E_{\text {out }}}{E_{\text {in }}}= & \left(1-\alpha_{0} L\right) \\
& +S L \alpha_{0} C \frac{W T_{2} \arctan \left(W T_{2} / 2\right)-\ln \left[1+\left(W T_{2} / 2\right)^{2}\right]}{W} .
\end{aligned}
$$

Equation (4) has also been derived with a different approach, namely directly solving the optical Bloch equations for an inhomogeneously broadened set of two-level systems in the limit of $W T_{1} \gg 1$ and large inhomogeneous broadening. Equation (4) predicts a linear increase of the transmission versus the spectral energy $S$ with a slope that depends on $W$ and $T_{2}$. In the inset of Fig. 1 the slopes obtained by a linear fit of the initial bleaching are shown, together with a fit according to Eq. (4) with $C$ and $T_{2}$ as free parameters, giving $T_{2}=290 \pm 80$ fs. The solid curves in Fig. 1 are calculated from Eq. (4) using the experimental values of $W$ showing a good agreement between model and experiment.

The FWM measurement is based on a heterodyne detection scheme, ${ }^{12}$ which allows for copolarized and copropagating pump and probe pulses. This technique avoids the phasematching restriction associated with the spatial selection geometry $^{2}$ and enables the use of long waveguides to enhance the FWM signal. The FWM signal created by two exciting pulses with time delays with respect to a reference pulse: $-t-\tau$ and $-t$, and frequency shifts $\omega_{1,2}$, respectively, is detected by its interference with the reference pulse at a frequency of $2 \omega_{2}-\omega_{1}$ using a lock-in technique. The lock-in signal is proportional to the average FWM electric field amplitude during the reference pulse duration. The time-resolved FWM signal is measured as a function of $t$ and $\tau$. The exciting and reference pulses were chosen as the shortest achievable from the laser source (140 fs). The measured FWM amplitude is shown in the left side of Fig. 3 as a function of $t$ for various $\tau$. The time shift of the signal with $\tau$ clearly indicates the photon-echo nature of the FWM. In the upper graph, the intensity of pulse 2 was around $0.2 \mathrm{pJ}$ 


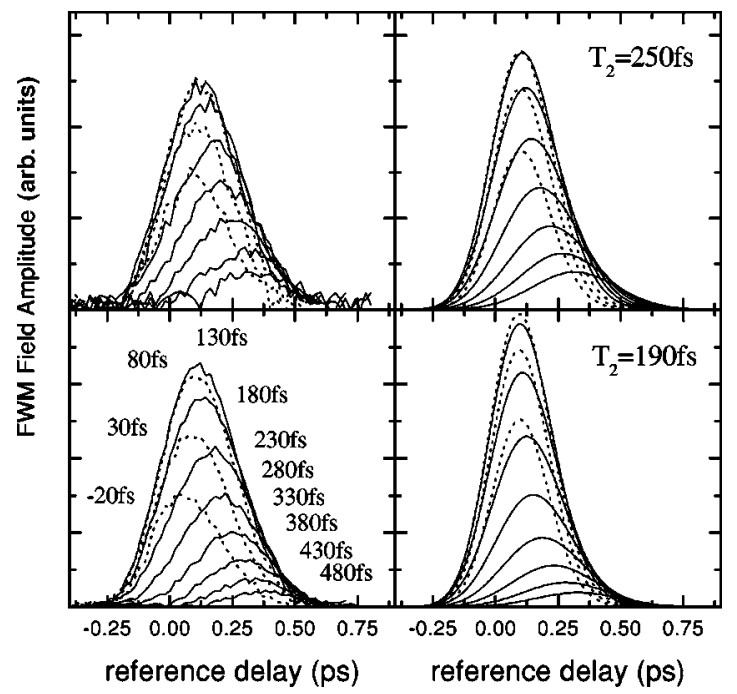

FIG. 3. Experimental (left) and calculated (right) time-resolved FWM electric field amplitudes for different delay times $\tau$ of the two exciting beams. The traces for delays $\tau \leqslant 80$ fs are plotted as dotted lines. The lower part of the figure refers to an excitation intensity twice the one in the upper part. The $T_{2}$ times used in the calculation are indicated, $T_{1}=5$ ps was determined by pump-probe experiments, and the pulse intensity FWHM of 140 fs was measured in their autocorrelation.

per pulse, twice the intensity of pulse 1 , and in the region of small absorption bleaching; the data in the lower graph were obtained with twice the excitation intensities.

In order to analyze the results, we have numerically calculated the third-order polarization by solving the optical Bloch equations for a two-level model, assuming Gaussian excitation pulses and an inhomogeneous broadening much broader than the spectral width of the pulse. The calculated electric field amplitude of the FWM is then convoluted with a Gaussian reference pulse to simulate our detection scheme. The resulting signal amplitudes are shown on the right side of Fig. 3 and the corresponding $T_{2}$ times are indicated. The simulations contain qualitatively all the features of the experimental data, like the initial increase of the signal maximum for increasing $\tau$, not expected for delta-pulses,${ }^{13}$ and the time shift of the signal for long delay times. Also, the simulations are in quantitative agreement with the measurements at long delay times. The time-integrated FWM field amplitude is shown in Fig. 4. The calculated values (dotted lines) are in good agreement with the experimental data (squares), with a small discrepancy for small delay times in the high excitation intensity case (upper curve). The $T_{2}$ time obtained by the calculations also coincides within error bars with the one obtained from the exponential fit of the data at long delay time (solid lines in Fig. 4). Both values are in agreement with the results of the SI-SHB within error bars.

In order to comment on the physical origin of the measured dephasing time we first summarize results reported in literature on dephasing in bulk and quantum well systems. The temperature dependence of the homogeneous linewidth at the excitonic transition in GaAs bulk and GaAs/ $/ \mathrm{Al}_{0.3} \mathrm{Ga}_{0.7}$ As quantum wells (QW's) up to room temperature (RT) was reported by Gammon et al. ${ }^{14}$ Comparison between QW's of different thicknesses demonstrate an LOphonon induced broadening weakly dependent on well

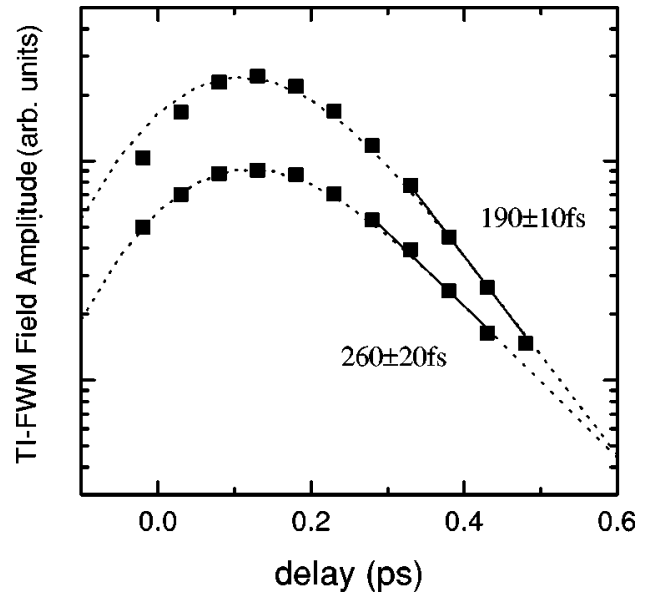

FIG. 4. Experimental (closed square) and calculated (dotted line) time-integrated FWM field amplitude for the two excitation intensities used in Fig. 3. Solid lines are exponential fits for long delay times; the corresponding dephasing times are indicated.

width, and comparable to the GaAs bulk value. At room temperature, $7 \mathrm{meV}$ FWHM line-width is measured in a 20 $\mathrm{nm}$ QW, dominated by LO-phonon absorption. A slightly larger value $(10 \mathrm{meV})$ is measured in bulk and attributed to a larger acoustic phonon broadening. A homogeneous linewidth of $7 \mathrm{meV}$ is equivalent to $T_{2}=190$ fs. Above the excitonic transition, faster dephasing times (less than $100 \mathrm{fs}$ ) of band-to-band transitions have been measured both in bulk and QW's, ${ }^{15-17}$ strongly influenced by carrier-carrier scattering, even though phonon emission gives additional dephasing. Dephasing times in bulk and QW diode lasers are generally considered to be fast (30-70 fs) due to carrier-carrier scattering, as also shown by the fast spectral-hole burning recovery time from pump-probe measurements in semiconductor optical amplifiers. ${ }^{18,19}$ Our results indicate a dephasing time from the ground-state excitonic transition slightly exceeding the ones measured by Gammon et al. ${ }^{14}$ at RT on QW's. This result is not surprising since the LO-phonon absorption is not strongly affected by the reduced dimensionality in these dots, because of the large number of closely spaced hole levels. Even if calculations of the energy level structure in the investigated binary-ternary QD's are missing due to the lack of precise information on the dot shape, calculations on InAs/GaAs dots of similar sizes show multiple hole levels with separations close to the LO-phonon energy. ${ }^{20}$ It has been recently shown ${ }^{21}$ that hole-phonon interaction is responsible for the lack of a "phonon bottleneck" for relaxation in $\operatorname{In}_{x} \mathrm{Ga}_{1-x} \mathrm{As} / \mathrm{GaAs}$ quantum dots even at $10 \mathrm{~K}$. As discussed by Gammon et al. ${ }^{14}$ the presence of multiple levels as final states in the LO-phonon absorption leads to an LO-phonon interaction weakly dependent on the confinement, in agreement with our findings.

We have observed that the intensity of the measured FWM signal follows a third-order behavior within the excitation intensity range discussed, while at higher excitation intensities higher-order effects appear. Within the third-order response, we observe only a weak intensity dependence of $T_{2}$. This indicates a weak exciton-exciton scattering when exciting only the lowest quantum dot transition. However, for an applied bias current for which the dot ground state is 
inverted, the FWM measurements show a dephasing time of below $70 \mathrm{fs}$, indicating a fast carrier-carrier scattering between carriers within a dot, once higher sublevels are occupied and several excitons are present. Such fast dephasing time implies a significant broadening of the DOS, reducing the expected superior values of differential gain and threshold current in QD lasers at RT compared to the ideal deltalike DOS. However, it also implies that the laser behavior can be dominated by the homogeneous broadening, resulting in single mode lasing very useful for $\mathrm{cw}$ applications, as also recently reported. ${ }^{22}$
In conclusion, we have presented the first measurements on the room temperature dephasing in InAs $/ \mathrm{In}_{x} \mathrm{Ga}_{1-x} \mathrm{As} / \mathrm{GaAs}$ self-assembled quantum dots. A $T_{2}$ only weakly dependent on the excitation density, with a low density value of $290 \pm 80$ fs from spectal-hole burning and of $260 \pm 20$ fs from four-wave mixing, is found. This is compared to reports in literature on bulk and QW structures and mainly attributed to LO-phonon absorption by the holes.

Parts of this work were supported by the Danish Technical Research Council in the framework of SCOOP and by DFG in the frame work of SFB 296.
${ }^{1}$ R. Loudon, The Quantum Theory of Light (Oxford Science Publications, Oxford, 1983).

${ }^{2}$ J. Shah, in Ultrafast Spectroscopy of Semiconductors and Semiconductor Nanostructures (Springer, Berlin, 1996), Chap. 2.

${ }^{3}$ D. Bimberg, M. Grundmann, and N.N. Ledentsov, Quantum Dot Heterostructures (Wiley, Chichester, 1999).

${ }^{4}$ A. Kuther, M. Bayer, A. Forchel, A. Gorbunov, V. B. Timofeev, F. Schäfer, and J. P. Reithmaier, Phys. Rev. B 58, R7508 (1998).

${ }^{5}$ N. H. Bonadeo, Gang Chen, D. Gammon, D. S. Katzer, D. Park, and D. G. Steel, Phys. Rev. Lett. 81, 2759 (1998).

${ }^{6}$ D. Gammon, E. S. Snow, B. V. Shanabrook, D. S. Katzer, and D. Park, Science 273, 87 (1996).

${ }^{7}$ D. Bimberg, N. Kristaedter, N. N. Ledensov, Z. I. Alferov, P. S. Kop'ev, and V. M. Ustinov, IEEE J. Sel. Top. Quantum Electron. 3, 196 (1997).

${ }^{8}$ F. Heinrichsdorff, M.-H. Mao, N. Kirstaedter, A. Krost, D. Bimberg, A. O. Kosogov, and P. Werner, Appl. Phys. Lett. 71, 22 (1997).

${ }^{9}$ F. Heinrichsdorff, A. Krost, D. Bimberg, A. O. Kosogov, and P. Werner, Appl. Surf. Sci. 123/124, 725 (1998).

${ }^{10}$ W. Demtröder, Laser Spectroscopy (Springer, Berlin, 1991).

${ }^{11}$ K. Meissner, B. Fluegel, H. Giessen, G. Mohs, R. Binder, S. W. Koch, and N. Peyghambarian, Phys. Rev. B 50, 17647 (1994).

${ }^{12}$ A. Mecozzi, J. Mørk, and M. Hofmann, Opt. Lett. 21, 1017
(1996); M. Hofmann, S. D. Brorson, J. Mørk, and A. Mecozzi, Appl. Phys. Lett. 68, 3236 (1996).

${ }^{13}$ J. Erland, K. H. Pantke, V. Mizeikis, V. G. Lyssenko, and J. M. Hvam, Phys. Rev. B 50, 15047 (1994).

${ }^{14}$ D. Gammon, S. Rudin, T. L. Reinecke, D. S. Katzer, and C. S. Kyono, Phys. Rev. B 51, 16785 (1995).

${ }^{15}$ P. C. Becker, H. L. Fragnito, C. H. Brito Cruz, R. L. Fork, J. E. Cunningham, J. E. Henry, and C. V. Shank, Phys. Rev. Lett. 61, 1647 (1988).

${ }^{16}$ T. Elsaesser, J. Shah, L. Rota, and P. Lugli, Phys. Rev. Lett. 66, 1757 (1991).

${ }^{17}$ J.-Y. Bigot, M. T. Portella, R. W. Schoenlein, J. E. Cunningham, and C. V. Shank, Phys. Rev. Lett. 67, 636 (1991).

${ }^{18}$ J. Mark and J. Mørk, Appl. Phys. Lett. 61, 2281 (1992); K. L. Hall, G. Lenz, A. M. Darwish, and E. P. Ippen, Opt. Commun. 111, 589 (1994).

${ }^{19}$ C.-K. Sun, B. Golubovic, J. G. Fujimoto, H. K. Choi, and C. A. Wang, Opt. Lett. 20, 210 (1995).

${ }^{20}$ M. Grundmann, O. Stier, and D. Bimberg, Phys. Rev. B 52, 11 969 (1995); O. Stier, M. Grundmann, and D. Bimberg, ibid. 59, 5688 (1999).

${ }^{21}$ T. S. Sosnowski, T. B. Norris, H. Jiang, J. Singh, K. Kamath, and P. Bhattacharya, Phys. Rev. B 57, R9423 (1998).

${ }^{22}$ M. Sugawara, K. Mukai, and Y. Nakata, Appl. Phys. Lett. 74, 1561 (1999). 